\title{
Two geometric constants for operators acting on a separable Banach space
}

\author{
E. Martin, E. Indurain, A. Plans and A. Rodes
}

ABSTRACT. The main result of this paper is the following: A separable Banach space $X$ is reflexive if and only if the infimum of the Gelfand numbers of any bounded linear operator defined on $X$ can be computed by means of just one sequence of nested, closed, finite codimensional subspaces with null intersection.

\section{INTRODUCTION}

Let $A$ be an operator from a separable Banach space $X$ into another Banach space $Y$. For every Markushevich basis of $X,\left(a_{n}\right)$, we define two numbers $h_{A,\left(a_{n}\right)}, H_{A,\left(a_{n}\right)}$, which give some geometrical insight about the space $X$ and also the operator $A$. In fact reflexivity of $X$ is characterized by stability of $H_{A,\left(a_{n}\right)}$ through changes of the $M$-basis, for every operator $A$, as theorem 1 states.

In the framework of reflexive Banach spaces these constants will be denoted simply $H_{A}$ and $h_{A}$ and they coincide respectively with the infimum of the Gelfand numbers of $A$, and with a precise lower bound of the Bernstein numbers of $A$ defined by Zemanek in [11]. So we come to the conclusion stated in theorem 2, that the infimum of the Gelfand numbers can be computed by means of a nested sequence of closed finite codimensional subspaces of null intersection.

In the third part we relate these numbers also with the spectral properties of $A$. Finally we see that for the particular case of a Hilbert space $X$, they are exactly the maximum and the minimum of the limit points of the spectrum of $\left(A^{*} A\right)^{1 / 2}$.

1980 Mathematics Subject Classification (1985 revision): 47A10, 47A53, 46B10, $40 \mathrm{~A} 05$.

Editorial de la Universidad Complutense. Madrid, 1988. 


\section{NOTATIONS, DEFINITIONS AND REMARKS}

We denote a Banach space by letters such as $X, Y$. The symbol [] will stand for closed linear span, and the symbol $\stackrel{m}{\rightarrow}$ for weak convergence. An $M$ basis will be the short name of a Markushevich basis, i.e. a fundamental sequence $\left(a_{n}\right)$ in a separable Banach space $X$, for which there exists another sequence $\left(a_{n}^{*}\right)$ in the dual $X^{*}$, called the conjugate sequence, with $a_{m}^{*}\left(a_{n}\right)=\delta_{m n}$ and such that $a_{n}^{*}(x)=0 \forall n \in N$, implies $x=0$. It is a well known result that $M$ bases always exist in a separable Banach space.

An $M$-basis $\left(a_{n}\right)$ of $X$ is called shrinking if for every $f$ of $X^{*}$

$$
\left\|\left.f\right|_{\left[a_{n}, \ldots\right]}\right\| \underset{n \rightarrow \infty}{\rightarrow \rightarrow 0}
$$

James has proven that this condition is equivalent to completeness of $\left(a_{n}^{*}\right)$ in $X^{*}$, that is $\left[\left(a_{n}^{*}\right)\right]=X^{*}$.

The set of all bounded linear operators from $X$ into $Y$ will be called $B(X, Y)$, and $\Phi_{+}(X, Y)$ will stand for the set of semi-Fredholm + operators, i.e. those bounded linear operators with closed range and finite dimensional kernel.

1. Let $X$ be a separable Banach space, $A \in B(X, Y)$ and let $\left(a_{n}\right)$ be an $M$ basis of $X$. We define the two numbers:

and

$$
H_{A,\left\{a_{n}\right\}}=\underset{n}{\left.\inf || A \mid \mathfrak{z}_{n, \ldots} \ldots\right] \mid} \mid
$$

$$
h_{A,\left(a_{n}\right\}}=\sup _{n} m\left(A \mid\left[a_{n} \ldots\right]\right)
$$

where $m$ stands for minimum modulus, i.e.

$$
m(A)=\inf _{\|x\|=1}\|A x\|
$$

The first of these numbers leads us to a characterization of reflexivity of $X$, as can be seen in

Theorem 1. The following statements are equivalent:

1. $X$ is reflexive.

2. For every bounded linear operator from $X$ into an arbitrary Banach space $Y, H_{A_{1}}\left\langle a_{n}\right)$ is independent of the M-basis $\left(a_{n}\right)$.

3. For every $f \in X^{*}, H_{f,\left(a_{n}\right)}$ is independent of the $M$-basis $\left(a_{n}\right)$.

Before giving the proof of the theorem we state three lemmas, which are easy to prove. 
Lemma 1. If $X$ is reflexive and separable, $\left(a_{n}\right)$ an $M$-basis of $X$ and $x_{n} \in\left[a_{n}, \ldots\right]$ with $\left\|x_{n}\right\|=1$, then $x_{n} \stackrel{w}{\rightarrow} 0$.

Lemma 2. Let $\left(x_{n}\right)$ be a normalized sequence in a Banach space $X$. Then $x_{n} \stackrel{w}{\rightarrow} 0$ if and only if for every finite codimensional, closed subspace $E$, there exists $\left(x_{n}^{\prime}\right)$ in $E$, with $x_{n}^{\prime}{ }^{w} \rightarrow 0,\left\|x_{n}^{\prime}\right\|=1$ and $\left\|x_{n}-x_{n}^{\prime}\right\| \rightarrow 0$.

It is a well known result, due to Zippin, that a Banach space $X$ with a basis is reflexive, if and only if every basis of $X$ is shrinking. The following lemma is a slight modification of this, not requiring the space to have a basis.

Lemma 3. A separable Banach space $X$ is reflexive if and only if every $M$ basis of $X$ in shrinking.

Proof. If $X$ is reflexive a closed subspace $F$ of $X^{*}$ which is total on $X$ must be the whole of $X^{*}$. Thus, for every $M$-basis $\left(a_{n}\right),\left[\left(a_{n}^{*}\right)\right]=X^{*}$ and equivalently $\left(a_{n}\right)$ is shrinking.

Conversely, if $X$ is non reflexive two cases arise. First, if $X^{*}$ is non separable, no $M$-basis can be shrinking. Second, if $X^{*}$ is separable, by the result of Gaposkin and Kadets, (see [4] p. 120), for every total subspace $F$ of $X^{*}$ there exists an $M$-basis $\left(a_{n}\right)$ of $X$ such that $\left[\left(a_{n}^{*}\right)\right]=F$. Thus $\left(a_{n}\right)$ is a non shrinking $M$-basis.

\section{Proof of theorem 1}

1) $\rightarrow 2$ ) Let $\left(a_{n}\right),\left(b_{n}\right)$ be two different normalized, $M$-bases of $X$. Denote by $\mu_{n}=\left\|\left.A\right|_{\left[a_{n+1}, a_{n+1}, \ldots\right]}\right\|$. Clearly $\mu_{n} \geqslant \mu_{n+1} \geqslant \cdots \geqslant 0$ and analogously $\mu_{n}^{\prime}=\left\|\left.A\right|_{\left[b_{n}, \ldots\right]}\right\|$.

Let $\left(\varepsilon_{n}\right)$ be a decreasing sequence of real numbers with $\varepsilon_{n} \rightarrow 0$. Choose for every $n, x_{n} \in\left[a_{n}, \ldots\right]$ such that $\left\|x_{n}\right\|=1$ and $\left|\mu_{n}-\left\|A x_{n}\right\|\right|<\varepsilon_{n}$. By lemma 1 , $x_{n} \stackrel{w}{\rightarrow} 0$. For a fixed $p \in N$, take into account the descomposition $X=\left[b_{1}, \ldots\right.$, $\left.b_{p-1}\right] \oplus\left[b_{p}, \ldots\right]$ and choose $\left(x_{n}^{\prime}\right)$ in $\left[b_{p}, \ldots\right]$, such that $\left\|x_{n}^{\prime}\right\|=1$ and $\left\|x_{n}-x_{n}^{\prime}\right\| \rightarrow 0$.

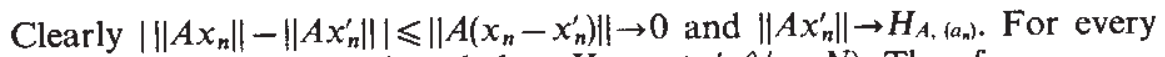
$n \in N,\left\|A x_{n}^{\prime}\right\| \leqslant\left\|\left.A\right|_{\left.b_{p}, \ldots\right]}\right\|=\mu_{p}^{\prime}$ and thus $H_{A,\left(a_{a}\right)} \leqslant \mu_{p}^{\prime}(\forall p \in N)$. Therefore

$$
H_{A,\left(a_{n}\right)} \leqslant \underset{p}{\inf \mu_{p}^{\prime}}=H_{A,\left(b_{n}\right)}
$$

Changing the roles of $\left(a_{n}\right)$ and $\left(b_{n}\right)$, the converse inequality is obtained, so $H_{A_{1}\left(a_{n}\right)}=H_{A_{1}\left(b_{n}\right)}$, which we will call $H_{A}$.

$2 \rightarrow 3$ is obvious. 
$3 \rightarrow 1$ Assume $X$ non reflexive. By lemma 3 there is a non shrinking $M$ basis $\left(a_{n}\right)$ in $X$. Take $g \notin\left[\left(a_{n}^{*}\right)\right]$ and take a biorthogonal system $\left(c_{n}, c_{n}^{*}\right)$ with

$\left[\left(c_{n}\right)_{n=1}^{\infty}\right]=\operatorname{Ker} \mathrm{g}$ and $\left(c_{n}^{*}\right)$ total on Ker g. Choose then $b_{1} \in X$ such that $g\left(b_{1}\right)=1$ and $b_{n}=c_{n-1}$ for $n \geqslant 2$. Now $\left(b_{n}\right)$ is an $M$ basis of $X$ with conjugate system $\left(b_{n}^{*}\right)$ given by $b_{1}^{*}=g$ and $b_{n+1}^{*}=c_{n}^{*}-c_{n}^{*}\left(b_{1}\right) g, n \geqslant 2$. Since $g \in\left[\left(b_{n}^{*}\right)\right]$, $H_{g,\left(b_{k}\right)}=0$. On the other hand $g \notin\left[\left(a_{n}^{*}\right)\right]$, which implies $H_{g,\left(a_{k}\right)} \neq 0$. Thus $H_{\theta,\left(b_{n}\right)} \neq H_{g,\left(a_{n}\right)}$.

Remark 1. A parallel to the theorem, setting $h_{A_{1}\left(a_{n}\right)}$ instead of $H_{A,\left(a_{n}\right),}$ does not hold. Although 1$) \rightarrow 2$ ) could be proved with the same argument, in 3 ) $\rightarrow$ ) we would obtain $h_{f,\left(a_{n}\right)}=0$ for every $\left(a_{n}\right)$, not depending on reflexivity of $X$.

Remark 2. Recall the following result of Milman and Tumarkin: «For a separable Banach space $X$ and a sequence $\left(E_{n}\right)$ of closed subspaces of $X$ such that $\operatorname{codim} E_{n}=n, E_{n} \supset E_{n+1} \forall n \in N$, and $n_{n} E_{n}=\{0\}$, there exists a biorthogonal system $\left(a_{n}, a_{n}^{*}\right)$ such that $\left.\left[a_{n+1}, a_{n+2}, \ldots\right]=E_{n}\right)$. (see [4], thm 1.6). According to it, theorem 1 could be stated without reference to an $M$-basis, only with a sequence of nested subspaces of finite codimension and null intersection.

From now on we abreviate $h_{A}$ and $H_{A}$ for $h_{A,\left(a_{A}\right)}$ and $H_{A_{3}\left(a_{A}\right)}$ respectively, taking for granted that we are dealing with a separable, reflexive, Banach space $X$.

2. We relate now $h_{A}$ and $H_{A}$ with $s$-numbers and some characteristics given by Zemanek in [11]. First recall that the Gelfand numbers of an operator $A$ are $\mathcal{c}_{n}(A)=\inf \{\| A|w|$, codim $W<n\}$, and that the Bernstein numbers are $u_{n}(A)=\sup \{m(A \mid w), \operatorname{dim} W \geqslant n\}$.

The sequences $c_{n}(A)$ and $u_{n}(A)$ are decreasing, bounded from below by

and

$$
c(A)=\inf \{\| A|w| \mid, \operatorname{codim} W<\infty\}
$$

$$
u(A)=\sup \{m(A \mid w), \operatorname{dim} W=\infty\}
$$

respectively. Also note that

and

$$
G(A)=\inf \{\|A \mid w\|, \operatorname{dim} W=\infty\} \leqslant c(A)
$$

$$
B(A)=\sup \left\{m\left(\left.A\right|_{w}\right), \operatorname{codim} W<\infty\right\} \leqslant u(A)
$$

In the quoted paper Zemanek obtains nice results concerning these numbers, and Kolmogorof numbers, Mityagin numbers, and their infima; he 
compares them with each other and with geometrical concepts such as SemiFredholm radius, reduced minimum modulus, etc. In the frame of separable reflexive Banach spaces we relate them with $h_{A}$ and $H_{A}$ as follows:

Proposition 1. Let $X$ be a separable reflexive Banach space and $A$ an operator from $X$ into any Banach space $Y$. Then $H_{A}=c(A)$ and $h_{A}=B(A)$.

Proof. Fix an $M$-basis $\left(a_{n}\right)$ and define as before $\left.\mu_{n}=\|A\|_{\left[u_{n}\right.} \ldots\right] \|$. Since $c_{n}(A)$ $=\inf \{\|A \mid w\|, \operatorname{codim} W<n\}$, it follows that $c_{n}(A) \leqslant \mu_{n}, \forall n \in N$. Therefore

$$
c(A)=\inf _{n} c_{n}(A) \leqslant \inf \mu_{n}=H_{A}
$$

For the converse inequality, fix a real number $\varepsilon>0$. Take $K \in N$ such that $c_{K}(A)<c(A)+\varepsilon$ and take also a subspace $W$ with $\operatorname{codim} W<k$ and $\| A|W|<c(A)+\varepsilon$. Any $M$-basis of $W$ can be extended in order to obtain an $M$ basis of $X$ (see [4] thm. 1.8). Thus, assume $\left(a_{n}\right)_{n=k}^{\infty}$ is an $M$-basis of $W$ and extend it to an $M$-basis of $X,\left(a_{n}\right)_{n=1}^{\infty}$. We have $H_{A} \leqslant\left\|\left.A\right|_{\left[a_{t}, \ldots\right]}\right\|<c(A)+\varepsilon$. This implies $H_{A}<c(A)$, and so $H_{A}=c(A)$.

A similar argument proves $h_{A}=B(A)$.

Now we can rewrite theorem 1 as follows:

Theorem 2. A separable Banach space $X$ is reflexive if and only if for every decreasing sequence of closed subspaces $\left(E^{n}\right)$ such that

a) $n=\operatorname{codim} E^{n}$.

b) $\cap_{n} E^{n}=\{0\}$.

and for every operator $A$ from $X$ into another Banach space $Y$, inf $\left\|A \mid E^{n}\right\|=c(A)$, where $c(A)$ is the infimum of the Gelfand numbers of $A$.

Proof. Just take into account Remark 2, and Proposition 2.

We can say further that when $X$ is separable and reflexive, $B(A)$ can also be computed by means of a nested sequence of closed subspaces satisfying the same conditions as those of theorem 2 .

Open questions. 1) What can be said when separability of $X$ is dropped?

2) Why does reflexivity establish such a difference?

3. We relate these constants now with spectral theory. The real numbers of the interval $\left[h_{A}, H_{A}\right]$ can be characterized as follows:

Theorem 3. Let $X$ be a separable reflexive Banach space, $A$ an operator defined from $X$ into an arbitrary Banach space $Y$. Then $\lambda \in\left[h_{A}, H_{A}\right]$ if and only if there exists $\left(x_{n}\right)$ in $X$, with $\left\|x_{n}\right\|=1, x_{n}{ }^{w} \rightarrow 0$, such that $\left\|A x_{n}\right\| \rightarrow \lambda$. 
Proof. Let $\lambda \in\left(h_{A}, H_{A}\right)$ and let $\left(a_{n}\right)$ be an $M$-basis of $X$. For every $n \in N$, let $v_{n}=\inf \left\{\|A x\| ; x \in\left[a_{n}, \ldots\right],\|x\|=1\right\}$. Then $v_{n} \leqslant h_{A}<\lambda<H_{A} \leqslant \mu_{n}=\| A\left|\left[a_{n} \ldots\right]\right|$. Take now $z_{n}, y_{n} \in\left[a_{n}, \ldots\right]$, with $\left\|z_{n}\right\|=\left\|y_{n}\right\|=1$ such that $v_{n} \leqslant\left\|A z_{n}\right\|<\lambda$ and $\mu_{n} \geqslant\left\|A y_{n}\right\|>\lambda$. Because the unit sphere of $\left[a_{n}, \ldots\right]$ is connected, one can find $x_{n} \in\left[a_{n}, \ldots\right],\left\|x_{n}\right\|=1$ such that $\left\|A x_{n}\right\|=\lambda$. Iterating for $n \in N$ we obtain a sequence $\left(x_{n}\right)$, which by lemma 1 converges weakly to zero, and $\left\|A x_{n}\right\|=\lambda$.

Slight modifications in this argument give the proof for $\lambda=h_{A}$ and $\lambda=H_{A}$.

Conversely, let $x_{n} \stackrel{\omega}{\rightarrow} 0,\left\|x_{n}\right\|=1$ and $\left\|A x_{n}\right\| \rightarrow \lambda$.

By lemma 2, for every fixed $p \in N$ we can choose $\left(z_{n}^{(p)}\right)_{n=1}^{\infty} \subset\left[a_{p}, a_{p+1}, \ldots\right]$ with $\left\|z_{n}^{(p)}\right\|=1 \forall n \in N, z_{n}^{(p)} \underset{n \rightarrow \infty}{\stackrel{w}{\rightarrow} 0}$ and $\left\|x_{n}-z_{n}^{(p)}\right\| \rightarrow 0$ wich implies | $\left\|A z_{n}^{(p)}\right\|-\left\|A x_{n}\right\| \mid \rightarrow 0$. Thus $\| A z_{n}^{(p)} \rightarrow \lambda$.

Select now a diagonal sequence $\left(z_{n(p)}^{(p)}\right)_{n=1}^{\infty}$, with $\left\|A z_{n(p)}^{(p)}\right\| \rightarrow \lambda$.

For every $p \in N, v_{p} \leqslant\left\|A z_{n(p)}^{(p)}\right\| \leqslant \mu_{p}$. Therefore, $\lambda \in\left[h_{A}, H_{A}\right]$.

Equality of $h_{A}$ and $H_{A}$, for an operator satisfying the former conditions, is determined by:

Corollary 1. The following are equivalent:

1) $h_{A}=H_{A}=r$.

2) Every normalized sequence $\left(x_{n}\right)$ of $X$, with $x_{n} \stackrel{w}{\rightarrow}, 0$ verifies $\left\|A x_{n}\right\| \rightarrow r$.

3) For every normalized $\left(x_{n}\right) \subset X$ with $x_{n} \stackrel{w}{\rightarrow} 0,\left(\left\|A x_{n}\right\|\right)$ is convergent.

Corollary 2. The operator $A$ is compact if and only if $h_{A}=H_{A}=0$.

Corollary 3. The operator $A$ is semi-Fredholm + if and only if $h_{A}>0$.

We omit the proof of these corollaries which are straight-forward, and do the same with the following propositions.

Proposition 2. Let $A \in B(X, Y)$. The following properties hold:

1). $h_{A}$ and $H_{A}$ are stable through compact perturbations, i.e., $h_{A+K}=h_{A}$ and $H_{A+K}=H_{A}$, for every compact operator $K$.

2) If $A, T \in B(X, X)$

$H_{A T}<\|A\| H_{T}$

$h_{A T} \geqslant h_{A} h_{T}$

If $T \in \Phi_{+}, H_{A T} \leqslant H_{A} H_{T}$.

3) $H_{A+B} \leqslant H_{A}+H_{B}$ and $h_{A+B} \leqslant h_{A}+h_{B}$.

Proposition 3. Let $A \in B(X, Y)$. The following properties are equivalent: 
1) $\left\|\left.A\right|_{E}\right\|=\|A\|$ for every closed, linear finite-codimensional subspace $E$.

2) $\|A\|=H_{A}$.

3) There exists a normalized weakly null sequence, $\left(x_{n}\right)$, such that $\left\|A x_{n}\right\| \rightarrow\|A\|$.

\section{GEOMETRICAL MEANING OF THE CONSTANTS $h_{A}$ AND $H_{A}$ FOR A HILBERT SPACE OPERATOR $A$}

Now let $X$ be a separable Hilbert space; $A$ a selfadjoint operator on $X$, and $E$ the spectral measure of $A$. Recall that a number $\lambda$ is a limit point of the spectrum of $A, \sigma(A)$, iff $E(V)$ has infinite rank for every neighborhood $V$ of $\lambda$.

Theorem 4. If $A$ is a selfadjoint operator on $X, H_{A}$ and $h_{A}$ are respectively, the maximum and the minimum of the limit points of the spectrum of $A$.

Proof. Suppose $\lambda$ is a limit point. According to Weyl's result (s. [3], p. 266) there exists $\left(x_{n}\right)$ in $X,\left\|x_{n}\right\|=1 \quad x_{n} \stackrel{w}{\rightarrow} 0$ and $\left\|(A-\lambda I) x_{n}\right\| \rightarrow 0$. In particular, $\left\|A x_{n}\right\| \rightarrow \lambda$ and therefore $h_{A} \leqslant \lambda \leqslant H_{A}$, although obviously not all the points of $\left[h_{A}, H_{A}\right]$ are limit points. Taking into account that addition of a self adjoint compact operator does not change the limit points on the spectrum, and by the results of Weyl and Von Neumann (s. [3], th. 2.1 p. 253), without loss of generality, we assume $A$ is a diagonal operator (obviously referred to an orthonormal basis of eigenvectors $\left(e_{n}\right)$ ), say

$$
A=\left[\begin{array}{llll}
\lambda_{1} & \ldots & 0 & \ldots \\
0 & \ldots & \lambda_{n} & \ldots \\
\ldots & \ldots & \ldots
\end{array}\right] \quad \lambda_{i} \geqslant 0 . \forall i \in N
$$

Let $\mu_{n}=\sup _{x \in\left[e_{0},\right]}\|A x\|$.

$$
\begin{aligned}
& x \in\left[e_{1}\right. \\
& \|x\|
\end{aligned}
$$

Clearly $\mu_{n}=\sup _{i>n} \lambda_{i}$

Choose now $\left(\lambda_{p_{n}}\right) \subset\left(\lambda_{n}\right)$ such that $\mu_{n}-\lambda_{p_{n}} \rightarrow 0$. We have $\lambda_{p_{n}} \rightarrow H_{A}$.

The fact $A e_{p_{n}}=\lambda_{p_{x}} e_{p_{n}}, \forall n \in N$, implies $\left\|\left(A-H_{A}\right) e_{p_{s} .}\right\| \rightarrow 0$, and therefore $H_{A}$ is a limit point, actually the largest of them. $A$ similar argument proves that $h_{A}$ is the minimum of the limit points of $A$.

Remark 3. If $A$ is not selfadjoint then $H_{A}$ and $h_{A}$ are not necessarily limit points of $\sigma(A)$. Nevertheless it can be easily proved, by polar descomposition and theorem 2 , that $h_{A}$ and $H_{A}$ are exactly the maximum and the minimum of the limit points of $\left(A^{*} A\right)^{1 / 2}$.

Remark 4. Other geometric properties of operators $A$ with $h_{A}=H_{A}$, can be 
seen in [7] and [8], where such operators are called "asymptotic similarities», because of their geometric behaviour.

Acknowledgements. The first author is indebted to Professor J. Zemanek for valuable conversations at Georgenthal, during the 8th-GDR-PolishSeminar on Banach Spaces, where part of this paper was presented as a talk.

\section{References}

[1] BeSSAGA, C., and PELCZYNSKY, A.: On bases and unconditional convergence of series in Banach spaces. Studia Math. T. XVII, 151-164 (1958).

[2] Diestel, J.: Sequences and series in Banach spaces. Springer Verlag, 1984.

[3] Kato, T.: Perturbation theory for linear operators. Springer Verlag, 1966.

[4] Milman, V.: Geometric theory of Banach spaces. Russian Math. Survey, 25, $111-170(1970)$.

[5] PIETSCH, A.: s-numbers of operators in Banach spaces. Studia Math. LI, 201223 (1974).

[6] PLANS, A.: Zerlegung von Folgen in Hilbertraum in Heterogonalsysteme. Archiv der Math., X, 304-306 (1954).

[7] Plans, A.: Resultados acerca de una generalización de la semejanza en el espacio de Hilbert. Collect. Math. V, XIII, 3. ${ }^{\circ}$, 241-248 (1961).

[8] PLANS, A.: Los operadores acotados en relación con los sistemas asintóticamente ortogonales. Collect. Math. V, XV, 104-110 (1963).

[9] RIESZ, F., and SZ.-NAGY, B.: Lecons d'analyse fonctionnelle. Gauthier-Villars, 1974.

[10] SINGER, 1.: Basic sequences and reflexivity of Banach spaces. Amn of Math. 52, 512-527 (1950).

[11] ZEMANEK, J.: Geometric characteristics of semi-Fredholm operators and their asymptotic behaviour. Studia Math. LXXX, 219-234 (1984).

[12] ZIPPIN, M.: A remark on bases and reflexivity in Banach spaces. Israel $J$. Math. Vol. 6, 74-79 (1968). 